\title{
Which Management for Gunshot Chest Wounds in a War Zone Hospital?
}

\section{Quelle prise en charge pour les plaies thoraciques par armes à feu dans un hôpital en zone de guerre?}

\author{
Abdoulaye Maman Bachir a b (D); Adakal Ousseini a ; Rabiou Sani a ; Sani Rachid ${ }^{\text {a }}$ \\ a Département de chirurgie, FSS, UDDM-Maradi-Niger. ${ }^{\mathrm{b}}$ Service de Chirurgie, CHR-Diffa, Niger
}

\begin{abstract}
Introduction: Gunshot chest wounds are a life-threatening surgical emergency without immediate management. Methods: The study period is two years, covering cases admitted from June 2017 to May 2019. The surgical service of the regional hospital center (CHR) of Diffa in Niger is one of the main second-level referral structures where victims of the armed conflict that started in 2014 in the Lake Chad basin are referred and treated. Through this study, we report the preliminary results of the hospital management gunshot chest Results: Firearm-related chest wounds accounted for $9.22 \%$ of firearm injury admissions, interesting especially young man subjects. Penetrating thoracic lesions involved 14 patients, 12 of whom had high and medium-abundant effusion treated by thoracic drainage. Two patients had pulmonary contusion and a fatal tamponade pattern respectively. Conclusion: Emergency chest drainage is a first-line saving procedure in the treatment of thoracic wounds with firearms in armed conflict.
\end{abstract}

Keywords: War surgery; Thoracic trauma; Penetrating wound; Gunshot wound; Thoracic drainage.

\section{RESUME}

Introduction : Les plaies thoraciques par arme à feu constituent une urgence chirurgicale mettant en jeu le pronostic vital en l'absence d'une prise en charge immédiate. Méthodologie : La période d'étude couvrait les cas admis entre Juin 2017 et Mai 2019 soit une durée de deux (2) ans. Le service de chirurgie du centre hospitalier régional (CHR) de Diffa au Niger est l'une des principales structures de référence de second niveau où sont acheminées et traitées les victimes du conflit armé déclenché depuis 2014 dans le bassin du lac Tchad. A travers cette étude, nous rapportons, les résultats préliminaires de la prise en charge hospitalière des plaies thoraciques par arme à feu. Résultats : Les plaies thoraciques par arme à feu représentaient $9.22 \%$ des admissions pour lésion par arme à feu. Elles intéressaient les sujets jeunes de sexe masculin. Les lésions thoraciques pénétrantes concernaient 14 patients dont 12 avec épanchement de grande et moyenne abondance traités par drainage thoracique. Les deux autres patients présentaient respectivement une contusion pulmonaire et un tableau de tamponnade mortelle. Conclusion : Le drainage thoracique en urgence est un geste salvateur de première intention dans le traitement des plaies thoraciques par arme à feu en situation de conflit armé.

MOTS CLES : Chirurgie de guerre ; Traumatisme thoracique ; Plaie pénétrante; Plaie par arme à feu ; Drainage thoracique.

Correspondence: Abdoulaye Maman Bachir. Département de chirurgie, FSS, BP : 465 Maradi-Niger.

Email : bachmed@hotmail.fr

Copyright (C) 2021 Maman Bachir A et al. This is an open access article distributed under the Creative Commons Attribution 4.0 International, which permits unrestricted use, distribution, and reproduction in any medium, provided the original work is properly cited. 


\section{INTRODUCTION}

La prise en charge des blessés par arme à feu constitue l'une des principales activités des hôpitaux au contact des zones de conflit. Celui en cours dans le bassin du lac Tchad est décrit comme l'un des plus meurtriers en Afrique avec environ 50000 décès et plus de 500000 déplacés et réfugiés d'après un rapport de l'Office de Coordination des Actions Humanitaires au Niger en 2019 (OCHA). Il s'agit de lésions dont la topographie et l'étendue déterminent le pronostic fonctionnel respiratoire ou vital du patient. Les plaies thoraciques pénétrantes sont responsables de plus d'un cinquième des causes de décès non naturel [1-3]. La prise en charge de ces blessés, souvent polytraumatisés requière la pluridisciplinarité et se conçoit dans les pays développés ayant des centres spécialisés rompus [4-6]. Le drainage thoracique constitue le geste de base dans la prise en charge des traumatismes pénétrants pleuropulmonaires [7, 8]. Ce geste nécessite une surveillance armée comme décrite dans le management chirurgical non opératoire [9]. La complexité de la prise en charge des plaies thoraciques par arme à feu dans la littérature [10] nous a conduit à évaluer nos résultats.

\section{PATIENTS ET METHODES}

Il s'agissait d'une étude rétrospective portant sur les cas de plaies thoraciques pénétrantes par arme à feu pris en charge au service de chirurgie du centre hospitalier régional (CHR) de Diffa, hôpital de deuxième niveau disposant d'une équipe de chirurgie générale assisté d'un service d'imagerie disposant d'un échographe et d'une radiographie standard. Cette équipe assure la prise en charge des urgences liées au conflit en cours dans la région. La période d'étude couvrait les cas admis entre Juin 2017 et Mai 2019 soit une durée de deux ans. Nous avons inclus dans l'étude, les patients, civiles et militaires victimes de traumatismes thoraciques admis vivants, quel que soit l'âge. Les critères de non inclusion comprenaient les cas admis décédés, les cas avec des dossiers incomplets ou inexploitables. Une évaluation clinique comprenant l'analyse des signes généraux (pouls, TA, SpO2, FR, coloration des téguments et des muqueuses), des signes physiques respiratoires et aussi l'examen général à la recherche des lésions associées. Le bilan biologique et radiologique comprenait une numération formule sanguine, un groupage sanguin rhésus et une radiographie thoracique dans tous les cas (figure 1-3). Le débridement chirurgical économique des plaies thoraciques suivi de la fermeture immédiate par suture thoracique pariétale était la règle. L'antibioprophylaxie ainsi que la prophylaxie anti tétanique et l'administration d'antalgiques étaient systématiques. Les patients ayant bénéficié d'un drainage thoracique étaient assistés par la kinésie thérapie respiratoire.

\section{RESULTATS}

Nous avons enregistré 14 cas de plaies pénétrantes thoraciques sur un total de 152 cas de plaies par arme à feu soit une proportion de $9,21 \%$. Tous étaient de sexe masculin dont 8 civiles et 6 militaires. Le service des urgences a connu par trois des séries d'afflux massif de blessé de guerre au cours de la période d'étude avec une moyenne 23 admissions par afflux. Nous avons enregistré 5 cas de plaies thoraciques au cours de ces afflux. Pour les 9 patients restants, il s'agissait de cas sporadiques. L'âge moyen est de 24,43 ans avec des extrêmes allant de 18 à 47 ans. Le délai moyen d'admission était de 5,7 heures avec des extrêmes allant de 1 heure à 14 heures.

L'évaluation initiale clinique et paraclinique a permis de distinguer :

-2 cas avec instabilité hémodynamique et cyanose réfractaire à la réanimation

- 10 cas avec hémothorax et/ou pneumothorax de moyenne abondance (figure $1 \mathrm{~A}, 2 \mathrm{~A}$ )

-2 cas avec instabilité hémodynamique en rapport avec un hémothorax de grande abondance compensée.

- Les lésions associées sont des atteintes de parties molles des membres $(n=4)$; abdominale $(n=1)$, et cervicale superficielle $(n=1)$

Dans 12 cas, il s'agissait de plaies thoraciques avec orifice d'entrée et de sortie du projectile.
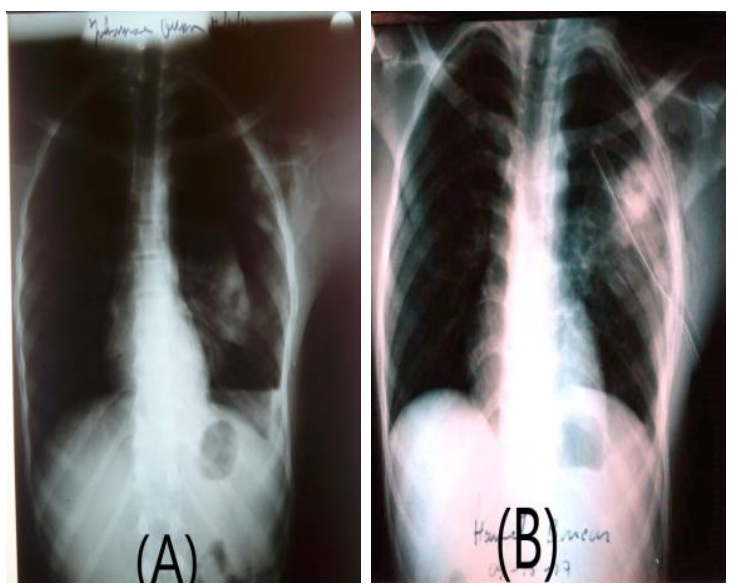

Figure 1 : (A) Radiographies thoracique standard de face montrant hémopneumothorax gauche (niveau hydro aérique) chez un patient victime de plaie de l'hémithorax gauche par balle. (B) Radiographies thoracique standard de face chez le même patient après drainage thoracique montrant le retour du poumon à la paroi avec le drain bien en place.
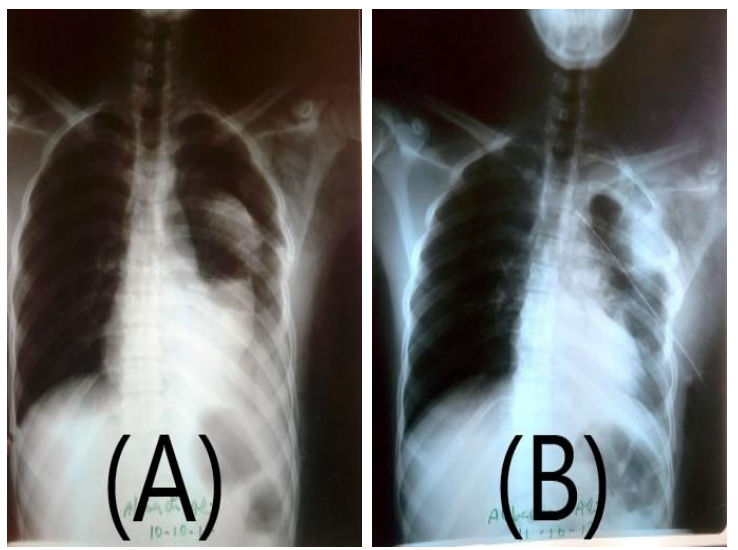

Figure 2: (A) Radiographies thoracique standard de face montrant hémopneumothorax gauche avec contusion pulmonaire suite à une plaie par balle. (B) Radiographies thoracique standard de contrôle après drainage thoracique montrant le retour du poumon à la paroi avec le drain bien en place, avec la contusion pulmonaire persistante en apico axillaire.

Le traitement avait consisté en un drainage thoracique chez 12 patients (figure 1B, 2B). L'extraction de projectile a concerné un patient de l'étude (figure 3). 


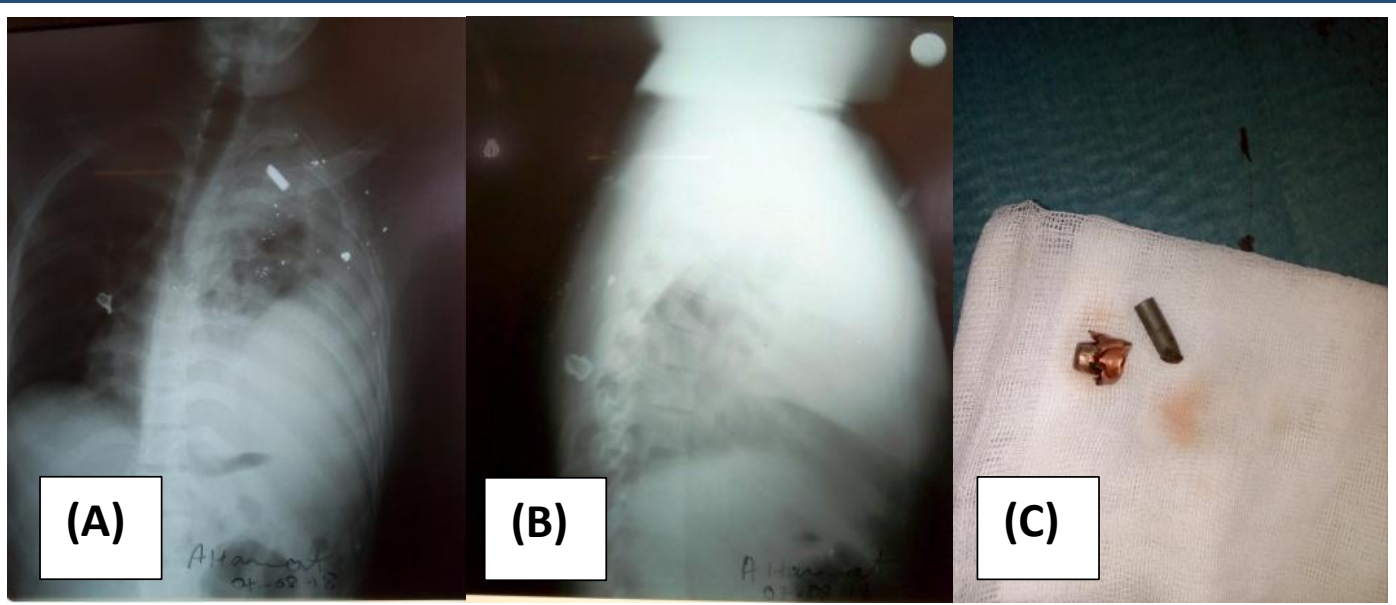

Figure 3 : (A) et (B) Radiographies thoracique standard, respectivement de face et de profil, montrant la présence des projectiles sur l'hémithorax gauche. (C) Projectiles après extraction.

L’oxygénothérapie au masque était administrée pour tous les cas présentant une détresse respiratoire $(n=10)$. La transfusion sanguine était réalisée chez 8 patients et 3 patients avaient bénéficié d'au moins 3 unités de sang. Les orifices pariétaux des plaies par balles étaient non délabrants et non soufflants ce qui a permis un parage et une fermeture primitive des plaies dans $100 \%$ des cas. Les suites étaient simples en ce qui concerne les 12 patients drainés avec des contrôles radio-cliniques satisfaisants. La durée moyenne de séjour était de 5,24 jours avec des extrêmes allant de 3 à 12 jours. Un patient présentant un hémothorax drainé avec bullage persistant a été référé vers un hôpital de référence disposant d'un service d'imagerie et de chirurgie thoracique. De même qu'un second patient parfaitement stable mais avec rétention de projectile dans l'aire cardiaque sur la radiographie thoracique. Deux cas de décès ont été enregistrés respectivement suite à une tamponnade et une contusion pulmonaire bilatérale.

\section{DISCUSSION-COMMENTAIRES}

Les plaies thoraciques par arme à feu sont relativement fréquentes dans le monde [11-13], représentant 9,21\% des plaies balistiques rencontrées en période de conflit d'après notre évaluation. Cette fréquence pouvant varier de 7 et $10 \%$ en fonction de la nature du conflit et surtout des moyens de ressuscitation disponibles en pré-hospitalier [4]. Elles intéressaient essentiellement les adultes jeunes de sexe masculin $[7,14]$ constituant l'essentiel des troupes combattantes. La prise en charge des plaies thoraciques par arme à feu nécessite un diagnostic certain ainsi que des procédures simples [15]. Les examens complémentaires sont hiérarchisés en fonction de l'état hémodynamique et respiratoire sans oublier la recherche des lésions associées notamment les atteintes des zones dites frontières [8], contrastant avec la pratique dans nos hôpitaux caractérisée par un plateau technique insuffisant voir obsolète ne disposant ni de scanner, ni d'échographie cardiaque, ni radiologue. Néanmoins, pour certains auteurs [9, 14], l'examen clinique aidé de la radiographie thoracique permettait une sélection des blessées avec d'une part ceux instables réfractaires à la réanimation et nécessitant une exploration chirurgicale et d'autre part les malades stables nécessitant une surveillance armée voire le transfert dans un centre spécialisé. Dans notre série, le drainage thoracique était efficace chez $100 \%$ des patients drainés. Il s'agit d'un geste essentiel de base qui doit être réalisé devant tout épanchement de moyenne ou grande abondance sous réserve de la maitrise chirurgical du geste et d'une surveillance du débit de drainage [7-9]. Le traitement des plaies thoraciques par arme à feu comporte des mesures générales mais aussi des gestes spécifiques en fonction des lésions. Concernant le traitement des lésions, deux attitudes nous semblant complémentaires sont proposées dans la littérature à savoir le management sélectif non opératoire $[9,14]$ et le management sélectif opératoire des plaies thoraciques [8]. Ainsi, en situation de guerre, les patients victimes de plaies thoraciques par arme à feu arrivant à l'hôpital stables ou stabilisés peuvent bénéficier d'un traitement conservateur par drainage thoracique [16]. La thoracotomie de ressuscitation peut constituer une alternative chez les patients instables [10, 16]. Les patients drainés et dont le débit de drainage est supérieur à $200 \mathrm{cc}$ par heure sur plus de 3 heures, ou dont le drain ramenait plus de 1500 cc d'emblée, ou en cas de bullage persistant devront bénéficier d'une thoracotomie en seconde intention en vue d'une hémostase et/ou une aérostase indispensable pour stabiliser le patient $[4,8,10]$. Les lésions de contusion pulmonaire sont les plus fréquemment retrouvées chez $50 \%$ des traumatisés thoraciques civils comme militaires $[17,18]$. Le diagnostic de certitude fait appel au scanner thoracique [19, 20]. Les complications infectieuses et/ou le Syndrome de Détresse Respiratoire aigu (SDRA) qui lui font suites sont responsables de la surmortalité observée dans ce groupe lésionnel [21, 22]. Les épanchements péricardiques minimes diagnostiqués par échographie cardiaque peuvent bénéficier d'un drainage péricardique en urgence. En cas de lésions spécifiques cardio-vasculaires diagnostiquées, seule une prise en charge en milieu spécialisée est indiquée [8].

\section{CONCLUSION}

La prise en charge initiale des plaies thoraciques par arme à feu peut être réalisée dans les hôpitaux de deuxième niveau disposant d'un service de chirurgie générale. D'où la nécessité de vulgariser la technique et de rendre disponible le matériel de drainage thoracique auprès des praticiens en chirurgie générale exerçant en milieu 
défavorisé. Les lésions spécifiques pleuro-pulmonaires et médiastinales graves doivent être adressées le plus tôt à des centres spécialisés.

\section{AUTHORS' CONTRIBUTIONS}

The participation of each author corresponds to the criteria of authorship and contributorship emphasized in the Recommendations for the Conduct, Reporting, Editing,

\section{REFERENCES}

[1] MacKenzie EJ. Epidemiology of injuries: current trends and future challenges. EpidemiolRev 2000; 2 2:112-119.

[2] LoCicero J 3rd, Mattox KL. Epidemiology of chest trauma. SurgClin North Am 1989; 69: 15-19.

[3] Von Oppell UO, Bautz P, de Groot M. Penetrating thoracic injuries: whathavelearnt. Thorac Cardiovasc Surg 2000; 4 8:55-61.

[4] Boddaert G, Mordant P, Le Pimpec-Barthes F, Martinod E, Aguir S, Leprince $\mathrm{P}$ et al. Surgical management of penetrating thoracic injuries during the Paris attacks on 13 November 2015. Eur J Cardiothorac Surg 2017;51:1195-202.

[5] Lerer LB, Knottenbelt JD (1994) Preventable mortality following sharp penetrating chest trauma. J Trauma 37(1):912

[6] Hardcastle TC, Finlayson M, van HM, Johnson B, Samuel C, Muckart DJ (2013) The prehospital burden of disease due totrauma in Kwa Zulu-Natal: the need for Afrocentric trauma systems. World J Surg 37(7):1513-1525.

[7] James Didier L. et al. Résultats de la prise en charge des plaies thoraciques à l'hôpital National de Niamey, Niger.RECAC 2017; 2(12): 19-24

[8] Andrew John N., Sorin E., Pradeep N. Selective operative management of penetrating chest Injuries. Curr Surg Rep (2019) 7:13

[9] Van Waes O. et al. Selective non operative management of penetrating thoracic injury. European Journal of Emergency Medicine 2016, Vol 00 No 00.

[10]Lesquen $\mathrm{H}$. et al. Challenges in war-related thoracic injury faced by French military surgeons in Afghanistan (20092013). Injury, Int. J. Care Injured 47 (2016) 1939-1944.

[11]Oparah SS, Mandal AK. Penetrating gunshot wounds of the chest in civilian practice: experience with 250 consecutive cases. Br J Surg 1978;65(January(1)):45-8. and Publication of Scholarly work in Medical Journalsof the International Committee of Medical Journal Editors. Indeed, all the authors have actively participated in the redaction, the revision of the manuscript and provided approval for this final revised version.

\section{COMPETING INTERESTS}

The authors declare no competing interests.

[12]Borja AR, Ransdell HT. Treatment of penetrating gunshot wounds of the chest. Experience with one hundred forty-five cases. Am J Surg 1971;122(July (1)):81-4.

[13] Muckart DJ, Meumann C, Botha JB. The changing pattern of penetrating torsotrauma in KwaZulu/Natal - a clinical andpathological review. S AfrMed J 1995;85 (November (11)):1172-4.

[14]Kong V. Y., Sartorius B., Clarke D. L. The selective conservative management of penetrating thoracic trauma is still appropriate in the currentera. Injury, Int. J. Care Injured 46 (2015) 49-53

[15]Dietrich D. et al. Penetrating thoracic trauma patients with gross physiological derangement: A responsibility for the general surgeon in the absence of trauma or cardiothoracic surgeon? World J Surg. Société Internationale de Chirurgie 2016. DOI 10.1007/s00268-016-3703-y.

[16]Siemens R. et al. Indications for thoracotomy following penetrating thoracic injury. The journal of trauma. Vol 17, No7, July $1977:$ 493-500.

[17]Boyd AD, Glassman LR. Trauma tothelung. Chest Surg Clin N Am 1997; 7:263-84.

[18] Keneally R, Szpisjak D. Thoracic trauma in Iraqand Afghanistan. J Trauma Acute Care Surg 2013; 74(5):1292-7.

[19]Durso A.M., Caban K., Munera F. Penetrating thoracic injury. Radiol Clin N Am 53 (2015) 675-693.

[20]Cerf C. Pulmonary contusions: physiopathology and management. Réanimation 16 (2007) 82-87.

[21] Michelet P, Couret D, Brégeon F, Perrin G, D'Journo XB, Pequignot V, Vig V, Auffray JP. Early onset pneumonia in severe chest trauma: a risk factor analysis. J Trauma. 2010; 68 (2) : 395-400

[22] Raghavendran K, Notter RH, Davidson BA, Helinski JD, Kunkel SL, Knight PR. Lung contusion: inflammatory mechanism sand interaction with other injuries. Shock 2009; 32:122-30. 